\title{
Wrist-worn wearables based on force myography: on the significance of user anthropometry
}

\author{
Mona Lisa Delva, Kim Lajoie [D, Mahta Khoshnam and Carlo Menon*
}

\author{
${ }^{*}$ Correspondence: \\ cmenon@sfu.ca \\ Menrva Research Group, \\ Schools of Mechatronic \\ Systems and Engineering \\ Science, Simon Fraser \\ University, Metro Vancouver, \\ Unit 250, 13450102 nd \\ Avenue, Surrey, BC V5A 156, \\ Canada
}

\begin{abstract}
Background: Force myography (FMG) is a non-invasive technology used to track functional movements and hand gestures by sensing volumetric changes in the limbs caused by muscle contraction. Force transmission through tissue implies that differences in tissue mechanics and/or architecture might impact FMG signal acquisition and the accuracy of gesture classifier models. The aim of this study is to identify if and how user anthropometry affects the quality of FMG signal acquisition and the performance of machine learning models trained to classify different hand and wrist gestures based on that data.
\end{abstract}

Methods: Wrist and forearm anthropometric measures were collected from a total of 21 volunteers aged between 22 and 82 years old. Participants performed a set of tasks while wearing a custom-designed FMG band. Primary outcome measure was the Spearman's correlation coefficient (R) between the anthropometric measures and FMG signal quality/ML model performance.

Results: Results demonstrated moderate $(0.3 \leq|R|<0.67)$ and strong $(0.67 \leq|R|)$ relationships for ratio of skinfold thickness to forearm circumference, grip strength and ratio of wrist to forearm circumference. These anthropometric features contributed to 23-30\% of the variability in FMG signal acquisition and as much as $50 \%$ of the variability in classification accuracy for single gestures.

Conclusions: Increased grip strength, larger forearm girth, and smaller skinfold-toforearm circumference ratio improve signal quality and gesture classification accuracy.

Keywords: Wearable technology, Force myography, User anthropometry, Biosignal quality, Machine learning

\section{Background}

Wearable technologies are now widely used to track and record movement related parameters [1]. For instance, electromyography (EMG) records muscle activity and force production occurring during movement, and can be used to provide the necessary commands for controlling external devices like computers [2, 3]. It is often used in rehabilitation protocols in research and in the clinic $[4,5]$. However, EMG requires proper skin preparation and electrode placement, making it difficult for the general public to use

(C) The Author(s) 2020. This article is licensed under a Creative Commons Attribution 4.0 International License, which permits use, sharing, adaptation, distribution and reproduction in any medium or format, as long as you give appropriate credit to the original author(s) and the source, provide a link to the Creative Commons licence, and indicate if changes were made. The images or other third party material in this article are included in the article's Creative Commons licence, unless indicated otherwise in a credit line to the material. If material is not included in the article's Creative Commons licence and your intended use is not permitted by statutory regulation or exceeds the permitted use, you will need to obtain permission directly from the copyright holder. To view a copy of this licence, visit http://creativecommons.org/ licenses/by/4.0/. The Creative Commons Public Domain Dedication waiver (http://creativecommons.org/publicdomain/zero/1.0/) applies to the data made available in this article, unless otherwise stated in a credit line to the data. 
in the home [6]. Inertial measurement units (IMUs) are small and able to detect limb acceleration, rotation and position [7], but are prone to signal noise and drift, as well as magnetic interference from home appliances and other devices [1]. Force myography (FMG), also referred to as topographic force mapping [8], residual kinetic imaging [9], and surface muscle pressure [10], records the volumetric changes that occur around a limb during muscle contraction. FMG has been used in multiple applications, including exoskeleton control [11-15], gait analysis [16], gesture identification [17-21], and rehabilitation [22, 23]. This technique has also been applied to assistive technology for amputees [11, 13] [24, 25] and stroke survivors [23]. Specifically, FMG does not require extensive skin preparation and specific electrode placement [8], nor does it require expertise for optimal implementation. Other advantages include increased signal stability over time for static gestures [12], robustness to external electrical interference and sweating [26], simpler signal processing than EMG datasets [17], and cost-effectiveness. These considerations are important for the deployment of FMG-based wearable devices in the general community.

Machine learning (ML) algorithms are recognized as powerful classification and pattern recognition tools to extract features from biosignals [27]. For example, FMG signals have been analyzed using ML-based techniques to monitor activities of both upper- and lower extremities for applications including discrete finger movement and hand gesture predictions [17, 18, 20,21, 28, 29], continuous finger movement predictions [30], and gait monitoring [16]. Linear discriminant analysis, support vector machine, and random forest algorithms are examples of such implemented methods.

Due to the nature of FMG signal acquisition, user anthropometry can lead to confounding effects. For instance, the pressure caused by muscle volumetric changes during contraction is transmitted to FMG sensors through overlying connective tissue, subcutaneous fat and skin. Intuitively speaking, the mechanical properties of each of these body components can affect signal quality. This is particularly significant, as numerous changes in body composition occur throughout the lifespan [31]. Specifically, declines in muscle mass and muscular strength [32] might affect how gestures are discriminated by FMG, as there is a positive correlation between muscle cross-sectional area and strength [33]. In addition, the amount of subcutaneous adipose tissue decreases with age [34], and connective tissue and skin become thinner and less elastic [35], all of which can influence how muscular forces are transmitted. Differences in body composition between biological males and females are also evident [36].

The aim of this preliminary study is to identify anthropometric measures that might systematically influence FMG signal acquisition and ML algorithm performance, and to quantify the extent of the variability introduced. This study is the first of its kind to consider the characteristics and effects unique to using FMG across as wide range of user characteristics.

\section{Methods}

\section{Participants}

Twenty-one participants (11 males and 10 females) were recruited from Simon Fraser University and the general community. Inclusion criteria were being able to follow instructions in English and perform the required gestures/tasks to completion. Exclusion 
criteria were self-identified neurological impairments and musculoskeletal barriers to functional movements. The study was approved by the Office of Research Ethics at Simon Fraser University, and all participants provided informed written consent. Testing took place at SFU and Confederation Seniors Centre in British Columbia, Canada.

\section{Anthropometric measures}

To investigate and identify the participant-specific measurable features that might cause variability in FMG signals, the following anthropometric measures were considered. Values obtained are reported in Table 1.

\section{Limb length and circumference}

Standard positioning for limb length and circumference measurements was a neutral shoulder, $90^{\circ}$ flexed elbow, neutral forearm, neutral wrist orientation, and relaxed hand. Forearm length was measured from the olecranon process to the ulnar styloid process and its circumference was measured at the widest part of the forearm. The length of the upper arm was measured from the acromial process to the olecranon process. Wrist circumference was measured within 1 inch proximal to the head of the radius and ulna, i.e., the thinnest part of the wrist. All measurements were done using a standard tape measure and were rounded to the nearest millimeter.

\section{Skinfold thickness}

Skinfold thickness was measured from the anterior aspect of the forearm, approximately at its widest part. Methods for taking skinfolds were taken from [42]. In brief, the skin was firmly grasped between the first three digits. The jaws of the calipers were then placed approximately $1 \mathrm{~cm}$ from where the skin was grasped, and the skin was released for measurement. An analog Slim Guide Skinfold Caliper (Creative Health Products, Ann Arbor, MI) was used for this purpose, and all measurements were rounded to the nearest millimeter.

Table 1 Participant demographics and anthropometric measures

\begin{tabular}{lc}
\hline Measure & Value \\
\hline Age $($ years) & $39.50(21.09)$ \\
Weight $(\mathrm{kg})$ & $78.06(14.64)$ \\
Height $(\mathrm{m})$ & $1.70(0.13)$ \\
Body mass index $\left(\mathrm{kg} / \mathrm{m}^{2}\right)$ & $26.81(4.25)$ \\
Wrist circumference $(\mathrm{cm})$ & $17.19(1.68)$ \\
Forearm circumference $(\mathrm{cm})$ & $26.09(3.24)$ \\
Forearm length $(\mathrm{cm})$ & $26.74(1.99)$ \\
Skinfold thickness $(\mathrm{cm})$ & $0.93(0.39)$ \\
Ratio: skinfold thickness to forearm circumference (unitless) & $0.03(0.02)$ \\
Ratio: wrist circumference to forearm circumference (unitless) & $0.67(0.07)$ \\
Maximum grip strength (kg) & $21.32(9.69)$ \\
\hline
\end{tabular}

Values are presented as $M\left(\sigma^{2}\right)$, where $\mu$ is the mean and $\sigma^{2}$ is the standard deviation 


\section{Active range of motion}

Active range of motion (ROM) of the wrist and the forearm were captured using a goniometer (Jamar Plus + Digital 8" Goniometer, Patterson Medical, Cedarburg, WI). Wrist ROM for flexion and extension was measured while the shoulder was in neutral position, with the elbow flexed at $90^{\circ}$ and the forearm held in neutral orientation. Participants were instructed to actively flex or extend their wrist to its fullest capacity, and measurements for each motion were taken as the smallest relative angle between the second meta-carpal and the central axis of the radius. Measurements were rounded to the nearest degree.

Forearm ROM for pronation and supination was measured while the shoulder was in neutral position, with the elbow flexed at $90^{\circ}$ and the wrist in neutral orientation. Participants were instructed to hold a pencil in a closed fist while actively pronating or supinating the forearm to its fullest capacity. Measurements for each motion were taken as the smallest relative angle between the starting and end positions of the length of the pencil. Measurements were rounded to the nearest degree.

\section{Grip strength}

Grip strength tests were performed with the shoulder and wrist in neutral positions and the elbow flexed at $90^{\circ}$. A non-deformable digital hand dynamometer (HD-BTA, Vernier Software \& Technology, Beaverton, OR) was used to measure grip strength. Participants were instructed to the hold the hand dynamometer in a closed fist and to squeeze it with maximum effort (maximum voluntary contraction) for approximately $3 \mathrm{~s}$. This test was repeated 3 times, and the average value was rounded to the nearest $0.01 \mathrm{~kg}$ [43].

All measurements were performed on the right side before the start of the protocol.

\section{Study protocol}

Participants were asked to use their right hand to perform instructed movements while seated on a chair of standard height and depth. This section describes the selected movements, the experimental task, and the procedure for data collection and analysis in detail.

\section{Selected movements}

Common hand gestures and wrist/forearm orientations for performing activities of daily living [44] were selected as the movement set performed by each participant. The selected hand gestures (Fig. 1 1-7) included:

1. Relax: fingers and thumb are not actively engaged in flexion or extension,

2. Open: fingers and thumb are fully extended and fully abducted,

3. Close: a fist with the buttressing of the distal tips of the phalanges against the central palm and buttressing of the thenar eminence and thumb against the dorsal surfaces of digits 2 and 3 [45] or the lateral aspect of the 2nd digit,

4. Point: only the 2nd digit (index finger) is fully extended, and the pad of the thumb is resting on the lateral aspect of the 3rd digit,

5. Key: the pad of the thumb is in contact with the proximal interphalangeal joint of the 2nd digit, 


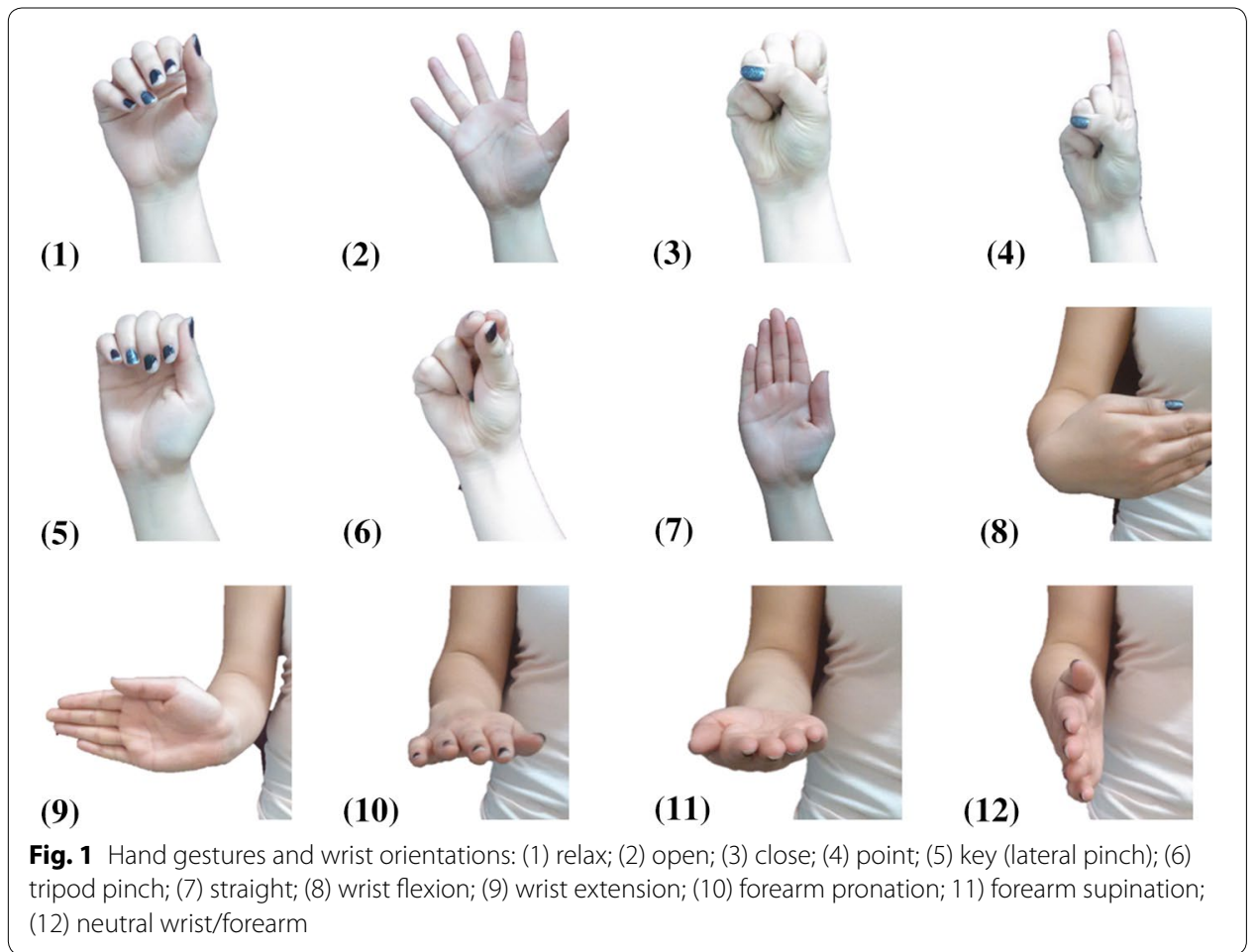

6. Tripod: the pads of the thumb, 2nd, and 3rd digits are in contact.

7. Straight: hand is open, and fingers and thumb are extended and adducted.

For each of the wrist and forearm orientations (flexion, extension, pronation, supination, and neutral), unless otherwise indicated, participants were instructed to keep the fingers and thumb fully extended and adducted (Fig. $18-12$ ). It is also worthwhile noting that the straight hand gesture (Fig. 17 ) and the neutral wrist/forearm orientations (Fig. 1 12) are similar. Therefore, when considering a combined set of hand gestures and wrist/forearm orientations, 11 distinct gestures were included.

To evaluate grip strength, participants were instructed to grip a hand dynamometer with the following grips: cylindrical, key and tripod (Fig. 2).

\section{Experimental task}

Three groups of tasks were defined based on the selected hand gestures and wrist/forearm orientations (Fig. 1):

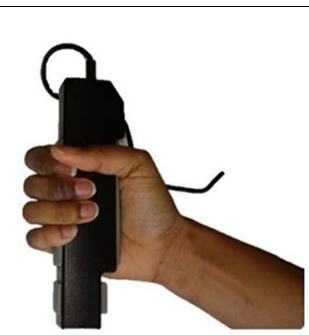

(1)

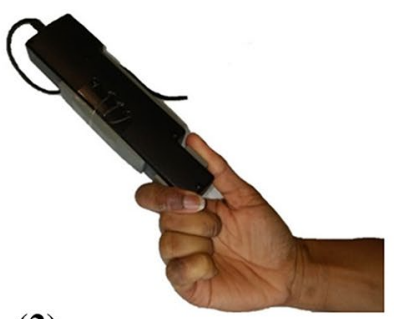

(2)

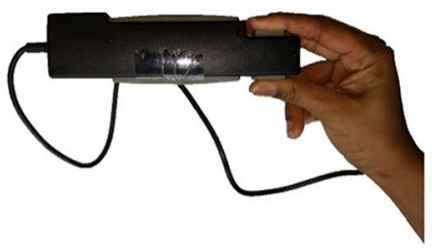

(3)

Fig. 2 Grip strength measurements: (1) cylindrical grip, (2) key grip, (3) tripod grip 
1. Static singleton gestures: participants were instructed to perform each of the hand gestures and wrist/forearm orientations individually, for a total of 11 different classes (gesture groups). Participants performed 5 randomized repetitions of each static gesture for approximately $7 \mathrm{~s}$ each.

2. Static compound gestures: participants were instructed to simultaneously perform a hand gesture and a wrist/forearm orientation. For example, for 'point_supination', participants were instructed to simultaneously point the hand (Fig. 14 ) and supinate the forearm (Fig. 1 11). Participants performed 5 randomized repetitions of each possible combination for approximately $7 \mathrm{~s}$ each.

3. Dynamic motions: participants were instructed to either move between two ROM extremes (i.e., from wrist flexion to extension), or to grip while applying minimal to maximal effort. Participants performed one repetition of each dynamic motion for 60 s. Table 2 provides an overview of the specific gestures that constitute each group of tasks.

Participants were in a relaxed posture before the performance of each gesture/motion.

\section{Data collection}

\section{Force myography (FMG) band}

A custom force myography (FMG) band was designed to quantify limb volumetric changes while performing the selected movements. Force-sensitive resistors (FSRs) were selected for FMG acquisition due to their low-profile dimensions, flexibility, costeffectiveness, wide-spread availability, and their ease of implementation into a portable and wireless device. The band used in this study utilized 16 FSRs (FSR 400, Interlink Electronics, Inc., Los Angeles, CA) in a staggered design. An FSR consists of a polymer thick film (PTF) circuitry printed on a flexible substrate with a variable force-resistance relationship. FSRs were implemented in series with a 4.6-k $\Omega$ resistor, and supplied with a voltage of 3.7 V. An ATMega328 microprocessor (Microchip Technology, Chandler, AZ) was used to facilitate data collection and transmission. Each FSR was sampled at approximately $10 \mathrm{~Hz}$, with raw analog values converted to a digital signal ranging from 0 to $1023(0.00361 \mathrm{~V} / \mathrm{bit})$. Digital values were time stamped and transmitted to an on-site computer via serial connection, then saved onto a .txt file for offline processing.

Table 2 Tasks completed by participants in experimental protocol

\begin{tabular}{llcl}
\hline Dynamic motions & Static singleton gestures & Static compound gestures \\
\hline Flexion $<->$ extension & Relax & All possible combinations of hand gestures and \\
Pronation<-> supination & Open & wrist/forearm orientations & Wrist orienta- \\
Cylindrical grip, squeeze and & Close & Hands gestures & tions \\
relax & Point & Relax & Flexion \\
Tripod grip, squeeze and & Key & Open & Extension \\
relax & Tripod & Close & Pronation \\
Key grip, squeeze and relax & Straight & Point & Supination \\
& Flexion & Key & Neutral \\
& Extension & Tripod & \\
& Pronation & & \\
\hline
\end{tabular}


The FSRs were backed with Flex foam and secured to the interior of the band, which was lined with cellulose acetate, a flexible and non-elastic material commonly used in overhead projector film transparencies. This material was used to facilitate a firmer contact between the FSR and the skin, while allowing the band to conform to the shape of the wrist. Participants donned the FMG band on the wrist, 1 to 1.5 in. proximal to the radial and ulnar styloid process surface landmarks. The placement of FSRs on the band is shown in Fig. 3 1. The placement of the band on the right wrist was similar to that shown in [46].

Instructions regarding the gestures were presented as images via a visual interface (Fig. 3 2) designed in LabVIEW 2014 and displayed in real time on a monitor positioned between eye level and desk level. When necessary, the investigator demonstrated the hand gesture desired at the time and/or corrected the participants' hand gesture to ensure uniformity between all participants.

\section{Data processing}

FMG signals were collected while participants performed instructed hand and wrist gestures (Table 2), then normalized based on the global minimum and maximum of acquired data. We then generated several ML models to establish trends of FMG behavior that were persistent despite selected models and chosen parameters. More specifically, four multi-output ML models, namely artificial neural network (ANN), extreme machine learning (ELM), linear discriminant analysis (LDA), and support vector machine (SVM), were generated and trained using built-in machine learning functions in MATLAB R2016a.

\section{Gesture classification using machine learning}

All ML models were trained using data collected while performing static singleton gestures (Table 2). These models previously demonstrated good accuracy in hand gesture classification based on FMG data [17, 47, 48]. ML models were then used to identify the singleton gestures performed during compound gestures or dynamic motions. For instance, during the 'Wrist Flexion/Extension' task, the model output is expected to be either flexion, neutral, or extension, based on the orientation of the hand with respect to the forearm. An exploratory analysis considering 11 static gestures (Table 2) as the

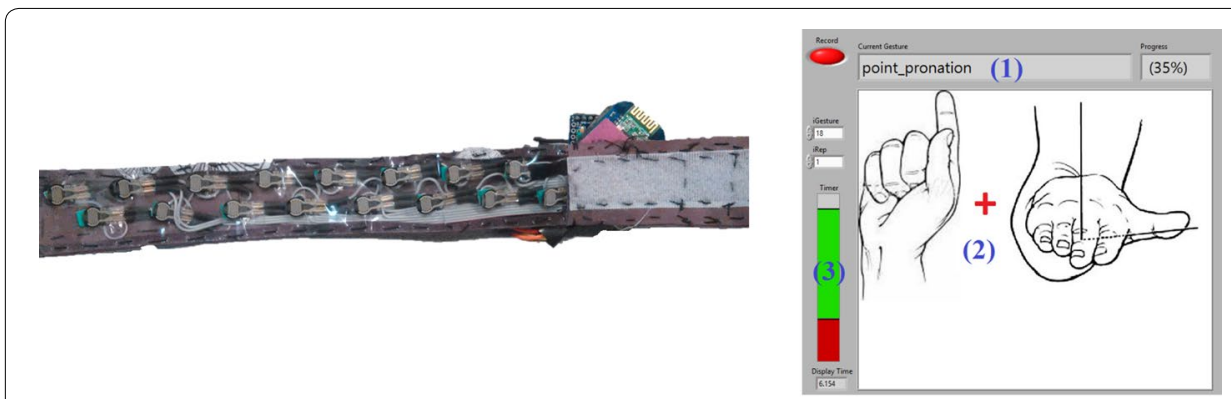

(1)

(2)

Fig. 3 (1) Staggered placement of the FSRs on a flexible non-elastic backing. (2) Instruction interface: (1) current instructed gesture, (2) instruction image, (3) gesture timer 
ML model output showed that training the ELM model using raw FMG data results in higher classification accuracy, while the classification accuracy of SVM, LDA, and ANN models is higher if normalized FMG data are used as the model input.

\section{Outcome measures}

The primary outcome measure for this study was the correlation between anthropometric measures ("Grip strength" section) and FMG signal quality/ML model performance. Spearman's correlation coefficient $(R)$ was used to evaluate such a correlation: a smaller $R$ value, where $|R|<0.33$, was considered a weak correlation, a medium range $R$ value, $0.33 \leq|R|<0.67$, represented a moderate correlation, and $0.67 \leq|\mathrm{R}|$ demonstrated a strong correlation.

Anthropometric measures considered were grip strength, skinfold thickness, and wrist and forearm circumferences. Moreover, several measurements were combined to create the following measures:

1. Ratio of skinfold thickness to forearm circumference (skinfold:forearm), which is an indicator of the ratio of bone/muscle to skin/adipose tissue and has a value in the range of $[0,1]$,

2. Ratio of wrist circumference to forearm circumference (wrist:forearm) which is an indicator of muscle bulk around the forearm and has a value in the range of $[0,1]$.

The quality of acquired FMG signals was quantified using two measures:

1. separability of gesture class clusters: to quantify the linear separateness of the data clusters used in classification, the separability index, $J_{B / W}$, was defined as

$$
J_{B / W}=\operatorname{trace}\left(S_{W}^{-1} S_{B}\right),
$$

where

$$
S_{W}=\sum_{i=1}^{n \text { Classes }} P_{i} S_{i} \text { and } S_{B}=\sum_{i=1}^{n \text { Classes }} P_{i}\left(m_{i}-m\right)\left(m_{i}-m\right)^{T}
$$

are within-class and between-class scatter matrices, respectively, with

I. $\omega_{i}$ : class label

II. $m_{i}$ : mean of class $\omega_{i}$

III. $K_{i}:$ number of samples in class $\omega_{i}$

IV. m: overall mean

V. $K$ : overall number of samples

VI. $P_{i}$ : the a priori probability of class $\omega_{i}=K_{i} / K$

VII. $S_{i}$ : scatter (covariance) matrix for class $\omega_{i}$

Intuitively, the optimal separability, i.e., larger values of $J_{B / W}$, is achieved by maximizing the between-class variance and minimizing the within-class variance. 
2. Sensitivity: this measure was defined as the magnitude of FMG response to incremental changes in orientation. Sensitivity was represented as the root mean square (RMS) of change in the FMG signal magnitude from baseline values across all sensors.

The performance of the developed machine learning models in detecting static and dynamic gestures was quantified using the following measures:

1. In the case of static gestures, the model performance was represented by accuracy, i.e., ratio of correctly classified gestures over total number of performed gestsures.

2. In the case of dynamic motions, the model performance was represented by the following measures: (1) the number of correct classes, (2) whether these classes overlapped with the training region. For instance, during the 'Wrist Flexion/Extension' task, the number of correct classes is three (flexion, neutral, and extension), and as long as the wrist is flexed, the output class is expected to be 'flexion'.

\section{Results}

\section{Participants' anthropometric measures and range of motion}

Anthropometric measures were determined for each participant as detailed in "Methods" section. Table 1 summarizes the average and standard deviation for those measurements.

During a number of dynamic motions, e.g., wrist flexion/extension, participants were asked to move through their full range of motion. The ROM values for different joints, measured at the beginning of the data collection session, are listed in Table 3.

Table 3 Range of motion (ROM) across all dynamic tasks

\begin{tabular}{lcc}
\hline & Female & Male \\
\hline Non-senior (19-59 years old) & & \\
Wrist flexion & $96.00^{\circ}\left(12.68^{\circ}\right)$ & $72.49^{\circ}\left(11.16^{\circ}\right)$ \\
Wrist extension & $-55.42^{\circ}\left(5.90^{\circ}\right)$ & $-55.40^{\circ}$ \\
& & $\left(11.95^{\circ}\right)$ \\
Forearm pronation & $59.68^{\circ}\left(22.74^{\circ}\right)$ & $70.00^{\circ}\left(13.80^{\circ}\right)$ \\
Forearm supination & $-64.62^{\circ}\left(5.66^{\circ}\right)$ & $-67.84^{\circ}$ \\
& & $\left(13.37^{\circ}\right)$ \\
Wrist full range & $151.42^{\circ}\left(10.80^{\circ}\right)$ & $127.90^{\circ}\left(16.62^{\circ}\right)$ \\
Forearm full range & $124.30^{\circ}\left(20.02^{\circ}\right)$ & $137.84^{\circ}\left(18.65^{\circ}\right)$ \\
Senior (60+years old) & & \\
Wrist flexion & $86.79^{\circ}\left(10.36^{\circ}\right)$ & $89.21^{\circ}\left(13.05^{\circ}\right)$ \\
Wrist extension & $-54.93^{\circ}\left(4.59^{\circ}\right)$ & $-51.12^{\circ}\left(9.32^{\circ}\right)$ \\
Forearm pronation & $82.21^{\circ}\left(14.18^{\circ}\right)$ & $72.51^{\circ}\left(10.69^{\circ}\right)$ \\
Forearm supination & $-60.20^{\circ}\left(14.15^{\circ}\right)$ & $-56.83^{\circ}$ \\
& & $\left(23.84^{\circ}\right)$ \\
Wrist full range & $141.72^{\circ}\left(14.66^{\circ}\right)$ & $140.33^{\circ}\left(3.73^{\circ}\right)$ \\
Forearm full range & $142.41^{\circ}\left(16.14^{\circ}\right)$ & $129.34^{\circ}\left(13.15^{\circ}\right)$
\end{tabular}

Positive ( + ) values for wrist flexion and forearm pronation, while using (-) values for wrist extension and forearm supination. Values are presented as $\mu\left(\sigma^{2}\right)$, where $\mu$ is the mean and $\sigma^{2}$ is the standard deviation 
Table 4 Correlation between anthropometric measures and separability of gesture classes

\begin{tabular}{lcc}
\hline Variable & \multicolumn{2}{l}{ Correlation coefficient (R) } \\
\cline { 2 - 3 } & Separability of hand classes & Separability of wrist classes \\
\hline Grip strength & 0.53 & 0.34 \\
Skinfold thickness & -0.20 & -0.16 \\
Skinfold:forearm & 0.57 & 0.40 \\
Wrist:forearm & -0.45 & -0.42 \\
Wrist circumference & -0.50 & -0.43 \\
Forearm circumference & -0.40 & -0.13 \\
\hline
\end{tabular}

Impact of anthropometric measures on FMG signal quality

Correlation between anthropometric measures and separability

At neutral wrist, the mean (standard deviation) separability of FMG clusters across the seven hand gestures (Fig. 1 1-7) was 124.79 (96.35).

With a straight hand gesture, the mean (standard deviation) separability of FMG clusters across the five wrist/forearm positions (Fig. $18-12$ ) was 76.21 (41.28). Mean correlations between user anthropometry and data cluster separability are tabulated in Table 4.

\section{Correlation between anthropometric measures and sensitivity}

Mean correlations between user anthropometry and sensitivity of acquired FMG signals are tabulated in Table 5. Grip strength and ratio of skinfold thickness to forearm circumference demonstrated the greatest relationship with FMG responsiveness.

Impact of anthropometric measures on the performance of ML models Correlation between anthropometric measures and model accuracy in classification of static gestures

To train the selected ML models, data collected from each participant were randomly split such that $60 \%$ of the collected data corresponding to each gesture were assigned to the training set, and the remaining $40 \%$ was used for testing the trained models. Mean training accuracies were 88.06, 90.38, 99.08, and 99.92 for SVM, LDA, ELM, and ANN, respectively. These models achieved mean testing classification accuracies of 87.95 , $89.96,98.89$, and 99.82 , respectively. It should be noted that an accuracy of $100 \%$ demonstrates perfect classification performance. Correlations between anthropometric measures and testing accuracy of considered ML models are tabulated in Table 6. Results

Table 5 Correlation between anthropometry and sensitivity

\begin{tabular}{lc}
\hline Variable & Correlation coefficient $(\boldsymbol{R})$ \\
\hline Grip strength & 0.55 \\
Skinfold thickness & -0.48 \\
Skinfold:forearm & -0.55 \\
Wrist:forearm & -0.39 \\
Wrist circumference & 0.13 \\
Forearm circumference & 0.33 \\
\hline
\end{tabular}


show that the accuracy of ELM and NN models weakly correlated with anthropometric measures. However, the skinfold thickness and ratio of skinfold thickness to forearm circumference highly affected the accuracy of SVM and LDA models, while other anthropometric measures had moderate-to-low correlations with the accuracy of these models.

\section{Correlation between anthropometric measures and model performance in classification of dynamic tasks}

The ML models trained for classification of static gestures were applied to the dynamic tasks presented in Table 2. Figure 4 shows the static gestures used to train models (blue box), non-static tasks onto which the trained models were applied (orange boxes), and the expected classification outputs based on the considered static gestures (gray boxes).

Figure 5 shows an example of the performance of trained models in identifying the dynamic wrist extension/flexion motion. In the shown case, the number of classes is three: flexion, neutral, extension (marked by number 1 on Fig. 5). The identified classes overlap with corresponding actual wrist orientations in regions where circular markers have the same color as the background shade (an example is marked by a purple circle numbered 2 on Fig. 5). Moreover, the variability within each cluster is characterized by the relative number of model outputs that did not correspond to the actual wrist orientation (an example is marked by a purple circle numbered 3 on Fig. 5).

Considering the data collected from all participants performing the dynamic tasks, Fig. 61 shows the accuracy of trained models in identifying the correct number of gestures for each task. Generally speaking, the correct number of gestures was correctly identified in at least $57 \%$ of cases across different tasks and trained models. Moreover, the number of gestures corresponding to dynamic wrist flexion/extension motion and forearm pronation/supination motion was correctly identified in more that $80 \%$ of cases using any of the trained models.

Figure 62 shows the proportion of correct overlap between the identified gestures and the corresponding actual orientation (training region) in wrist flexion/extension and forearm pronation/supination motions. Defining 'Extremes' as the fully flexed/extended state for the wrist and the fully pronated/supinated for the forearm, and 'Neutral' as the

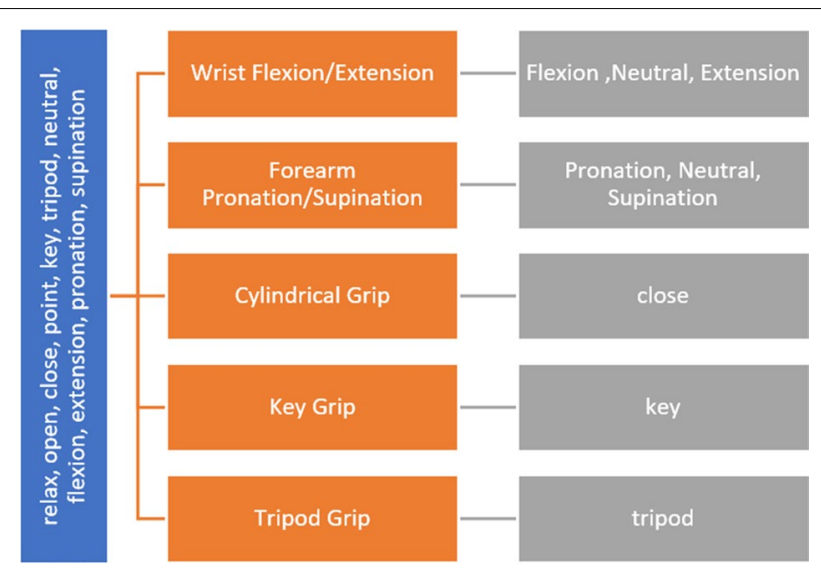

Fig. 4 Schema for exploring gesture identification during non-static activities 
Table 6 Correlation between anthropometric measures and gesture/orientation classification accuracy (SVM support vector machine, LDA linear discriminant analysis, ELM extreme learning machine, $N N$ neural network)

\begin{tabular}{lcccc}
\hline Variable & SVM & LDA & ELM & NN \\
\hline Grip strength & 0.44 & 0.50 & 0.18 & 0.24 \\
Skinfold thickness & -0.66 & -0.71 & -0.22 & -0.20 \\
Skinfold:forearm & -0.68 & -0.71 & -0.28 & -0.24 \\
Wrist:forearm & -0.57 & -0.46 & -0.23 & -0.28 \\
Wrist circumference & -0.14 & -0.11 & 0.03 & -0.03 \\
Forearm circumference & 0.23 & 0.13 & 0.17 & 0.12 \\
\hline
\end{tabular}

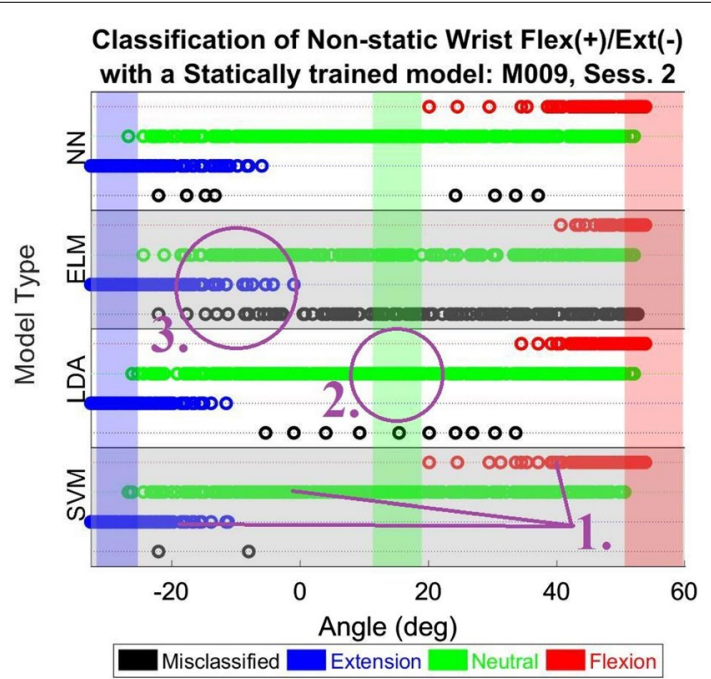

Fig. 5 Performance of the models trained with static gestures in classifying dynamic wrist motion. Circular markers indicate the output of each model. Positive $(+)$ values are assigned to wrist flexion and forearm pronation, while negative (-) values are assigned to wrist extension and forearm supination

neutral wrist and neutral forearm position, the percentage of correct overlap is higher in the 'Neutral', reaching 96\% using the trained SVM model. In the case of 'Extremes', SVM shows a high overlap percentage of $93 \%$.

Figure 63 shows that continuous identification of close, key, and tripod grips during dynamic cylindrical grip, key grip, and tripod grip tasks is quite poor. This is indicated by low correct overlap (between $11 \%$ and $28 \%$ ) between the identified gesture and its actual counterpart. Such a poor performance might be attributed to the participants' handling of an object (a hand dynamometer) in the dynamic tasks, which was not present when performing the static gestures

All model performance measures reported in this section had low correlations $(|R|<0.3)$ with anthropometric measures.

\section{Additional results}

Although this study was not focused on the effects of age and gender on the outcome measures, we investigated further. Specifically, participants were divided into two age 
Table 7 Details of participant demographics and anthropometric measures based on age and gender

\begin{tabular}{|c|c|c|c|c|}
\hline & \multicolumn{2}{|c|}{ Non-senior (19-59 years old) } & \multicolumn{2}{|c|}{ Senior $(60+$ years old $)$} \\
\hline & Female & Male & Female & Male \\
\hline Number & 6 & 9 & 4 & 2 \\
\hline Age (years) & $26.25(2.44)$ & $27.11(3.55)$ & $74.75(5.44)$ & $64.50(4.95)$ \\
\hline Weight (kg) & $65.40(14.71)$ & $87.11(9.25)$ & $74.50(15.51)$ & $82.41(3.41)$ \\
\hline Height (m) & $1.61(0.04)$ & $1.83(0.08)$ & $1.59(0.07)$ & $1.65(0.07)$ \\
\hline $\begin{array}{l}\text { Body mass index (kg/ } \\
\left.\mathrm{m}^{2}\right)\end{array}$ & $25.01(4.74)$ & $26.12(3.17)$ & $29.31(5.57)$ & $30.30(1.34)$ \\
\hline $\begin{array}{l}\text { Wrist circumference } \\
(\mathrm{cm})\end{array}$ & $15.92(1.88)$ & $17.72(0.97)$ & $16.88(1.80)$ & $19.25(0.35)$ \\
\hline $\begin{array}{l}\text { Forearm circumference } \\
(\mathrm{cm})\end{array}$ & $24.33(2.82)$ & $27.50(3.82)$ & $25.00(1.78)$ & $27.25(0.35)$ \\
\hline Forearm length (cm) & $25.17(1.57)$ & $27.89(1.54)$ & $25.63(1.80)$ & $28.50(0.71)$ \\
\hline $\begin{array}{l}\text { Skinfold THICKNESS } \\
(\mathrm{cm})\end{array}$ & $0.99(0.23)$ & $0.66(0.25)$ & $1.25(0.51)$ & $1.30(0.42)$ \\
\hline $\begin{array}{l}\text { Ratio: skinfold thick- } \\
\text { ness to forearm } \\
\text { circumference } \\
\text { (unitless) }\end{array}$ & $0.04(0.01)$ & $0.02(0.01)$ & $0.05(0.02)$ & $0.05(0.02)$ \\
\hline $\begin{array}{l}\text { Ratio: wrist circumfer- } \\
\text { ence to forearm } \\
\text { circumference } \\
\text { (unitless) }\end{array}$ & $0.65(0.04)$ & $0.66(0.10)$ & $0.68(0.05)$ & $0.71(0.02)$ \\
\hline $\begin{array}{l}\text { Maximum grip } \\
\text { strength (kg) }\end{array}$ & $16.11(4.43)$ & $29.83(7.55)$ & $10.54(2.72)$ & $20.24(4.45)$ \\
\hline
\end{tabular}

Values are presented as $\mu \sigma^{2}$ ), where $\mu$ is the mean and $\sigma^{2}$ is the standard deviation

groups, namely seniors (above 60 years old) and non-seniors (between 19 and 60 years old). Table 7 provides age- and gender-specific details of participant demographics and anthropometric measures. Student's $t$ test and ANOVA were implemented to reveal whether age and gender affect the quality of acquired FMG signals and the performance of the developed machine learning models. This analysis showed age-associated differences in the accuracy of classification, and in the separability of hand gestures and wrist gestures ( $p$-value $<0.005)$. However, it should be noted that the number of participants was not balanced in age and gender groups (Table 7). Therefore, these results might have been skewed. This aspect is addressed further in the Discussion.

\section{Discussion}

Force myography is a non-invasive method to measure volumetric changes in muscle cross-sectional area, which are transmitted through tissue. The resultant surface pressure changes can be used to track and identify movements and gestures. Due to the nature of force transduction through underlying tissue, user anthropometry may influence the quality of FMG signal acquisition and FMG-based modeling. Understanding how anthropometry can confound FMG performance is important for the creation of robust user-centered designs for wearable technologies. This exploratory study investigated the relationship between user anthropometric measures and FMG acquisition/ modeling, and further quantified its magnitude. 
With respect to FMG sensitivity, the greatest significant contributors to variability in FMG responsiveness were ratio of skinfold thickness to forearm circumference $(R=-0.55)$, and grip strength $(R=0.55)$. Grip strength is an anthropometric measure directly related to muscle fiber cross-sectional area. As FMG measures the volumetric changes that occur with activity, it is understandable that lower grip strengths would result in lower magnitudes of changes, which is supported by our results. When performing static gestures, increased grip strength was associated with improved hand gesture separability $(R=0.53)$. The relationship between grip strength and wrist motions was not as strong $(R=0.34)$. This difference is attributed to the muscle-tendon organization of the forearm and recruitment during wrist/hand movements. In the distal forearm, the anterior flexor compartment is composed of tendons responsible for finger flexion. Relative to motions of the wrist, these tendons are more engaged in the cylindrical grip during grip strength measurements, as well as in 5 of the 7 hand gestures, hence the stronger correlation. Despite the differences in the separability of wrist gestures versus hand gestures, the strong relationship between grip strength and gesture classification accuracy has implications for using FMG with certain populations.

Another variable related to muscle cross-sectional area and grip strength was the ratio of wrist to forearm circumference. Previous studies show a correlation between grip strength and forearm circumference [37, 38]. In the present study, the greater the forearm circumference relative to the wrist circumference, the better the improvements in sensitivity to change $(R=-0.39)$, which further highlights the significance of strength, as well as the underlying musculoskeletal bulk in FMG signal acquisition and gesture classification.

The correlation between the ratio of skin fold thickness to forearm circumference and FMG sensitivity had a moderate and negative correlation $(R=-0.55)$. As non-rigid structures, the skin and subcutaneous fat act as dampeners of the forces produced by muscle fibers as they are transmitted to the FSR sensors. Thus, an increase in adipose tissue likely increases the dampening effect on FMG signals and decrease the discriminability of gestures. This ratio also takes into consideration the combined effects of the skin/fat dampening and muscle cross-sectional area. A high ratio of skinfold thickness to forearm circumference also strongly correlated with lower classification accuracy, i.e.,

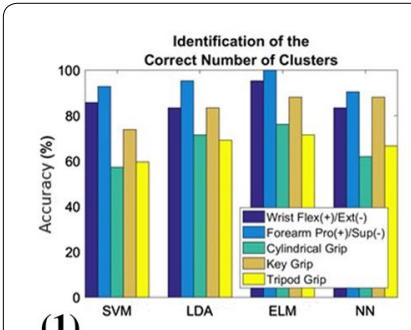

(1)

Fig. 6 (1) Accuracy of trained models in identifying the correct number of static gestures occurring during the dynamic tasks. (2) Proportion of correct overlap between identified gestures and actual arm/wrist orientation in wrist flexion/extension and forearm pronation/supination motions. (3) Proportion of correct overlap between identified gestures and corresponding actual gesture in cylindrical grip, key grip, and tripod grip during dynamic tasks (SVM support vector machine, LDA linear discriminant analysis, ELM extreme learning machine, NN neural network)

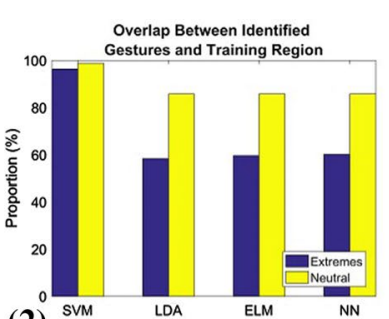

(2) ${ }^{\text {sin }}$

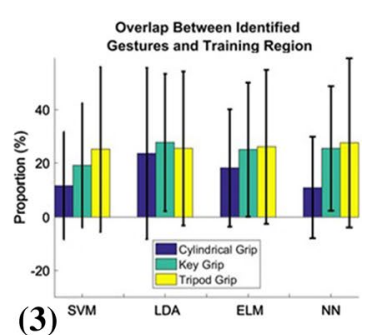

(3)

.


$R=-0.68$ and -0.71 for SVM and LDA models, respectively. Moderate relationships were observed with gesture separability for hand and wrist gestures, with $R=0.57$ and $R=0.40$, respectively.

Using models trained with static gestures to identify expected similar gestures performed during non-static activity presented moderate-to-high success (Fig. 6 1). However, this performance was not consistent throughout the full range of motion or effort and was not necessarily related to training conditions. Clusters of correctly identified activity were more consistent at the extremes of movements, but were least likely to overlap with the training region for that intended gesture. At neutral wrist/forearm, there was a high degree of variability and misclassification, however it is surmised that this is due to the inherently low separability of hand gestures. When the models were trained by performing hand gestures without objects, there was a low probability of correctly identifying that gesture in the presence of an object $(<27 \%)$. The performance of static models during non-static activity had little to no relationship with anthropometry measures and is therefore more likely related to the nature of the machine learning paradigm itself.

The identification of anthropometric measures that can influence FMG signal acquisition quality and modeling accuracy has the potential to enhance the development of FMG technology. It is recommended to explore these factors as weighting factors for classification and regression algorithms. In addition, this knowledge would be beneficial for sensor selection and calibration in future designs. However, the authors acknowledge that the current study has limitations. First, the number of participants is relatively low, and more participants would increase statistical power. In addition, the protocol was performed using the right arm, regardless of hand dominance. Grip strength has a documented relationship with hand dominance [39], which was not addressed in the current work. It is recommended that future iterations of this work be performed on both dominant and non-dominant hands and evaluate intra-subject variances.

The participants recruited for this study covered a wide age range (Table 7). The emphasis of this study was not on age and gender effects, although previous research reported a relationship between age, gender and weight, and anthropometry measures such as skinfold thickness [40]. Given the potential for FMG technology to be used as an assistive tool for senior populations [41], we thus encourage future studies to consider the effects of age and gender on the relationship between anthropometric measures and FMG signal acquisition quality and modeling accuracy.

\section{Conclusions}

We presented data and analysis identifying and quantifying how individual differences in anthropometry measures can affect the quality of FMG signal acquisition and FMGbased modeling. Sixteen force-sensing resistors were arranged as a grid in a portable and wearable band to track hand gestures and wrist/forearm orientations. Participants perform a pre-selected set of gestures commonly used to perform activities of daily living. The protocol as presented identified three key anthropometric measures, namely grip strength, ratio of wrist to forearm circumference, and ratio of skinfold thickness to forearm circumference, as having moderate $(0.3<|R|<0.6)$ influence on FMG signal acquisition and modeling. Increased grip strength, larger forearm girth, and smaller 
skinfold-to-forearm circumference ratio were shown to improve signal quality and hand gesture classification accuracy during static and dynamic conditions. These results have implications for the design of FMG technology for select populations like seniors, as well as guidelines for algorithm development. Future research could consider anthropometric characteristics as features in classification/regression algorithms and compare FMG to other myography modalities, while also considering the effect of age and gender.

\section{Abbreviations}

EMG: Electromyography; IMU: Inertial measurement unit; FMG: Force myography; ML: Machine learning; ROM: Range of motion; FSR: Force-sensitive resistors; PTF: Polymer thick film; ANN: Artificial neural network; ELM: Extreme machine learning; LDA: Linear discriminant analysis; SVM: Support vector machine; RMS: Root mean square.

\section{Acknowledgements}

The authors wish to acknowledge the members of the Menrva Research Group for their assistance with the project.

\section{Authors' contributions}

MLD performed the experiments, analyzed and interpreted the data, and wrote the manuscript first draft. $\mathrm{KL}$ and MK were major contributors in writing the manuscript. CM acquired funding and supervised the research project. All authors read and approved the final manuscript.

\section{Funding}

This work was supported by the Natural Sciences and Engineering Research Council of Canada (NSERC), the Canadian Institutes of Health Research (CIHR), and the Canada Research Chair (CRC) program.

\section{Availability of data and materials}

The datasets used and/or analyzed during the current study are available from the corresponding author on reasonable request.

\section{Ethics approval and consent to participate}

The Office of Research Ethics at Simon Fraser University approved the study, and all participants gave informed written consent.

\section{Consent for publication}

Not applicable.

\section{Competing interests}

$\mathrm{CM}$ and members of his research team have a vested interest in commercializing the technology tested in this study, if it is proven to be successful and may benefit financially from its potential commercialization. The data are readily available upon request.

Received: 23 March 2020 Accepted: 28 May 2020

Published online: 12 June 2020

\section{References}

1. Filippeschi A, Schmitz N, Miezal M, Bleser G, Ruffaldi E, Stricker D. Survey of motion tracking methods based on inertial sensors: a focus on upper limb human motion. Sensors. 2017;17(6):1257.

2. Choi C, Kim J. A real-time EMG-based assistive computer interface for the upper limb disabled. In: 2007 IEEE 10 th International Conference on Rehabilitation Robotics, ICORR'07. 2007. p. 459-62.

3. Katz D, Hafsia L Ben, Salem O, Mehaoua A. Intelligent remote control of smart home devices using physiological parameters. In: 2015 17th International Conference on E-Health Networking, Application and Services, HealthCom 2015. Institute of electrical and electronics engineers Inc.; 2015. p. 280-5.

4. Cesqui B, Tropea P, Micera S, Krebs HI. EMG-based pattern recognition approach in post stroke robot-aided rehabilitation: a feasibility study. J Neuroeng Rehabil. 2013;10(1):75.

5. Huang Y, Lai WP, Qian Q, Hu X, Tam EWC, Zheng Y. Translation of robot-assisted rehabilitation to clinical service: a comparison of the rehabilitation effectiveness of EMG-driven robot hand assisted upper limb training in practical clinical service and in clinical trial with laboratory configuration for chronic stroke. Biomed Eng Online. 2018;17(1):91.

6. Merletti R, Muceli S. Tutorial surface EMG detection in space and time: Best practices. J Electromyogr Kinesiol. 2019;49:102363.

7. Morrow MM, Lowndes BR, Fortune E, Kaufman KR, Susan Hallbeck M, Kern PE, et al. Validation of inertial measurement units for upper body kinematics. J Appl Biomech. 2017;33(3):227-32.

8. Castellini C, Artemiadis P, Wininger M, Ajoudani A, Alimusaj M, Bicchi A, et al. Proceedings of the first workshop on peripheral machine interfaces: going beyond traditional surface electromyography. Front Neurorobot. 2014;8(22):1-17.

9. Phillips SL, Craelius W. Residual kinetic imaging: a versatile interface for prosthetic control. Robotica. 2005;23(3):277-82. 
10. Yungher DA, Wininger MT, Barr JB, Craelius W, Threlkeld AJ. Surface muscle pressure as a measure of active and passive behavior of muscles during gait. Med Eng Phys. 2011;33(4):464-71.

11. Cho E, Chen R, Merhi L-K, Xiao Z, Pousett B, Menon C. Force myography to control robotic upper extremity prostheses: a feasibility study. Front Bioeng Biotechnol. 2016;4(March):1-12.

12. Connan M, Ramírez ER, Vodermayer B, Castellini C. Assessment of a wearable force and electromyography device and comparison of the related signals for myocontrol. Front Neurorobot. 2016;10:17.

13. Abboudi RL, Glass CA, Newby NA, Flint JA, Craelius W. A biomimetic controller for a multifinger prosthesis. IEEE Trans Rehabil Eng. 1999;7(2):121-9.

14. Xiao ZG, Elnady AM, Menon C. Control an exoskeleton for forearm rotation using FMG. In: Proceedings of the IEEE RAS and EMBS International Conference on Biomedical Robotics and Biomechatronics. IEEE Computer Society; 2014. p. 591-6.

15. Sangha S, Elnady AM, Menon C. A compact robotic orthosis for wrist assistance. In: Proceedings of the IEEE RAS and EMBS International Conference on Biomedical Robotics and Biomechatronics. IEEE Computer Society; 2016. p. $1080-5$.

16. Jiang $\mathrm{X}$, Chu KH, Khoshnam M, Menon C. A wearable gait phase detection system based on force myography techniques. Sensors. 2018;18(1279):1-13.

17. Jiang X, Merhi LK, Xiao ZG, Menon C. Exploration of force myography and surface electromyography in hand gesture classification. Med Eng Phys. 2017;41:63-73.

18. Xiao ZG, Menon C. Towards the development of a wearable feedback system for monitoring the activities of the upper-extremities. J Neuroeng Rehabil. 2014;11(1):2.

19. Yang D, Chhatre N, Campi F, Menon C. Feasibility of Support Vector Machine gesture classification on a wearable embedded device. In: Canadian Conference on Electrical and Computer Engineering. Institute of Electrical and Electronics Engineers Inc:; 2016.

20. Jiang X, Merhi LK, Menon C. Force exertion affects grasp classification using force myography. IEEE Transact Hum Mach Syst. 2017:48:1-8.

21. Li N, Yang D, Jiang L, Liu H, Cai H. Combined use of FSR sensor array and SVM classifier for finger motion recognition based on pressure distribution map. J Bionic Eng. 2012;9(1):39-47.

22. Sadarangani G. MC. A wearable sensor system for rehabilitation aplications. In: Proceedings of the 2015 IEEE International Conference on Rehabilitation Robotics (ICORR); Singapore. 2015. p. 672-7.

23. Sadarangani GP, Jiang X, Simpson LA, Eng JJ, Menon C. Force myography for monitoring grasping in individuals with stroke with mild to moderate upper-extremity impairments: a preliminary investigation in a controlled environment. Front Bioeng Biotechnol. 2017;5(July):1-11.

24. Rasouli M, Chellamuthu K, Cabibihan JJ, Kukreja SL. Towards enhanced control of upper prosthetic limbs: A forcemyographic approach. In: Proceedings of the IEEE RAS and EMBS International Conference on Biomedical Robotics and Biomechatronics. IEEE Computer Society; 2016. p. 232-6.

25. Radmand A, Scheme E, Englehart K. High-density force myography: a possible alternative for upper-limb prosthetic control. J Rehabil Res Dev. 2016;53(4):443-56.

26. Yaniger SI. Force Sensing resistors ${ }^{\mathrm{TM}}$ a review of the technology. In: Electro International, ELECTR 1991 - Conference Record. Institute of Electrical and Electronics Engineers Inc.; 1991. p. 666-8.

27. Supratak A, Wu C, Dong H, Sun K. GY. Survey on Feature Extraction and Applications of Biosignals. In: (eds) HA, editor. Machine Learning for Health Informatics Lecture Notes in Computer Science. Cham: Springer; 2016. p. 161-82.

28. Kadkhodayan A, Jiang X, Menon C. Continuous prediction of finger movements using force myography. J Med Biol Eng. 2016;36(4):594-604.

29. Sadarangani GP, Menon C. A preliminary investigation on the utility of temporal features of Force Myography in the two-class problem of grasp vs. no-grasp in the presence of upper-extremity movements. Biomed Eng Online. 2017;16(1):59.

30. Sadeghi Chegani R, Menon C. Regressing grasping using force myography: an exploratory study. Biomed Eng Online. 2018:17(1):159.

31. Kim S, Leng XI, Kritchevsky SB. Body composition and physical function in older adults with various comorbidities. Innov Aging. 2017. https://doi.org/10.1093/geroni/igx008.

32. Young JC. Physical Dimensions of Aging, 2nd edition. By Waneen W. Spirduso, Karen L. Francis, and Priscilla G. MacRae. Champaign, IL: Human Kinetics. Am J Hum Biol. 2006;18(1):156-8.

33. Jones EJ, Bishop PA, Woods AK, Green JM. Cross-sectional area and muscular strength. Sports Medicine. 2008;38(12):987-94

34. Sepe A, Tchkonia T, Thomou T, Zamboni M, Kirkland JL. Aging and regional differences in fat cell progenitors-a minireview. Gerontology. 201 1;57(1):66-75.

35. Fenske NA, Lober CW. Structural and functional changes of normal aging skin. J Am Acad Dermatol. 1986;15(4):571-85.

36. Wells JCK. Sexual dimorphism of body composition. Best Pract Res Clin Endocrinol Metab. 2007;21(3):415-30.

37. Abe T, Loenneke JP. Handgrip strength dominance is associated with difference in forearm muscle size. J Phys Ther Sci. 2015;27(7):2147-9.

38. Anakwe RE, Huntley JS, McEachan JE. Grip strength and forearm circumference in a healthy population. J Hand Surg Am. 2007;32(2):203-9.

39. Chatterjee S, Chowdhuri BJ. Comparison of grip strength and isomeric endurance between the right and left hands of men and their relationship with age and other physical parameters. J Hum Ergol. 1991;20(1):41-50.

40. Durnin JVGA, Womersley J. Body fat assessed from total body density and its estimation from skinfold thickness: measurements on 481 men and women aged from 16 to 72 Years. Br J Nutr. 1974;32(1):77-97.

41. Delva ML, Sakr M, Chegani RS, Khoshnam M, Menon C. Investigation into the potential to create a force myographybased smart-home controller for aging populations. Proc IEEE RAS EMBS Int Conf Biomed Robot Biomechatronics. 2018;2018-Augus:770-5.

42. Donoghue WC. How to measure your \% bodyfat. Seventh Ed. Creative Health Products; 2009. p. 22. 
43. Mathiowetz V, Kashman N, Volland G, Weber K, Dowe M, Rogers S. Grip and pinch strength: normative data for adults. Arch Phys Med Rehabil. 1985;66(2):69-74.

44. Peerdeman B, Boere D, Witteveen H, in't Veld R, Hermens H, Stramigioli S, et al. Myoelectric forearm prostheses: State of the art from a user-centered perspective. J Rehabil Res Dev. 2011;48(6):719-37.

45. Morgan MH, Carrier DR. Protective buttressing of the human fist and the evolution of hominin hands. J Exp Biol. 2013;216(2):236-44.

46. Delva ML, Menon C. FSR based force myography (FMG) stability throughout non-stationary upper extremity tasks. Futur Technol Conf. 2017;(November):891-6.

47. Xiao ZG, Menon C. A review of force myography research and development. Sensors. 2019;19(20):4557.

48. Xiao ZG, Menon C. Counting grasping action using force myography: an exploratory study with healthy individuals. JMIR Rehabil Assist Technol. 2017;4(1):1-17.

\section{Publisher's Note}

Springer Nature remains neutral with regard to jurisdictional claims in published maps and institutional affiliations.

- fast, convenient online submission

- thorough peer review by experienced researchers in your field

- rapid publication on acceptance

- support for research data, including large and complex data types

- gold Open Access which fosters wider collaboration and increased citations

- maximum visibility for your research: over 100M website views per year

At BMC, research is always in progress.

Learn more biomedcentral.com/submissions 